\title{
A Fast and Automatic Approach to Extract the Brain and Midsagittal Lines from FDG-PET Head Scans
}

\author{
Guoyu Qian ${ }^{1,2}$, Suhuai Luo ${ }^{1}$, Jesse Jin ${ }^{1}$, Mira Park ${ }^{1}$, Jiaming Li ${ }^{3}$, Wieslaw L. Nowinski ${ }^{2}$ \\ ${ }^{1}$ School of Design, Communication and IT, The University of Newcastle, Australia \\ ${ }^{2}$ Biomedical Imaging Lab, Agency for Science, Technology and Research, Singapore \\ ${ }^{3}$ Information \& Communication Technologies, CSIRO ICT Centre, Australia
}

\begin{abstract}
A fully automated approach is presented to extract brain areas efficiently from FDG-PET head scans. A threshold value is automatically calculated from the histogram graph of the brain images, followed by region growing and morphological operations, to segment brain areas from these images. Next, the midsagittal lines on axial slices are detected to separate the brain into two hemispheres. The proposed approach has been applied to 226 cases of normal controls and patients with neurological diseases. The average processing time is about 3 seconds on a standard personal computer. The experiment has shown promising results.
\end{abstract}

\section{Keywords-image processing; brain extraction; midsagittal line}

\section{INTRODUCTION}

Positron emission tomography (PET) is a powerful, noninvasive tool used to study the biochemistry and physiology of the living organs like human brain [1]. It is a nuclear medicine imaging technique to produce a three dimensional (3D) image of functional processes in the body. After a tracer is injected into a patient, a PET scanner can form images of the tracer distribution. Fluoro-deoxy-glucose (FDG) is a commonly used tracer to distinguish the high-glucose-consuming cells from the other parts of the body. Usually it is used for assessment of glucose metabolism mainly in the brain, heart, and kidney. This imaging technique is widely used for tumor detection and diagnosis of the neurological diseases, e.g. Alzheimer's disease (AD).

Identification of the brain volume from PET images is a basis for many applications, e.g. registration with the images of other modality like magnetic resonance imaging (MRI) or computed tomography (CT), quantitative analysis of brain cortex, etc. Due to the poor spatial resolution and the low signal-noise ratio of PET images, automated brain extraction from those images is still a challenging task, as well as the identification of a midsagittal line (MSL) which separates the brain image into two hemispheres.

Brain extraction from PET images can be manually done by an experienced physician or radiologist. Such a timeconsuming work produces a subjective and unrepeatable result. Alternatively, the registration tools such as Analyze [2] and Statistical Parametric Mapping (SPM) [3] are applied to extract physiological information from PET images by registration with a brain template or another structural modality (e.g. MRI) which has anatomical information to be used as a reference. These tools need the user to load images and operate several steps on them for registration. There are also a few automated methods of brain extraction from PET images including thresholding [4] that needs user interventions, cluster analysis and pattern classification methods $[5,6]$ which are sensitive to noise and patient movement during the study.

A separation plane, i.e. midsagittal plane (MSP), of two hemispheres in the brain is an important landmark for further volumetric symmetry analysis, such as registration, quantification, and pathology detection. It can be the first step to automatically detect the anterior commissure and posterior commissure [7], and other landmarks [8]. Those landmarks are used in functional neurosurgery [9], brain mapping [10], and other neuroradiology applications [11]. There are several methods to locate the MSP in MRI or CT images [12-15]. Those methods may not apply to PET images.

In this paper, a fully automated approach to extract two hemispheres of the brain from FDG-PET images is presented. Histogram graph, thresholding, region growing, and morphological operations are applied to extract the brain from a volumetric FDG-PET dataset. Our method, which was earlier successfully applied to extract the MSP from MR images, is extended to locate the MSL in PET images. A total of 226 cases of FDG PET images including normal controls, and patients with mild cognitive impairment (MCI) and $A D$, were tested. The proposed approach requires no user intervention, and needs only a few seconds processing time.

\section{MATERIALS AND METHOD}

\section{A. Materials}

The proposed approach of brain extraction and MSL detection was evaluated with the FDG-PET images from Alzheimer's Disease Neuroimaing Initiative (ADNI) database. Each case has 6 dynamic frames with scanning every 5 minutes from 30 to 60 minutes after FDG injection. Those scans were preprocessed by the following steps: 1) registered to the first frame, 2) averaged, 3) reoriented into a grid of $160 \times 160 \times 96$ voxels with voxel size $1.5 \times 1.5 \times 1.5 \mathrm{~mm}$ [16]. The preprocessed images have their horizontal axis paralleled with the anterior commissure and posterior commissure. Figure 1 shows the examples of images from a patient with MCI. Figure 1a is one slice of the original baseline images; Figure $1 \mathrm{~b}$ is another original image co-registered with baseline images; Figure 1c is an averaged image of 6 dynamic scans; and Figure $1 \mathrm{~d}$ is a slice after reorientation. 

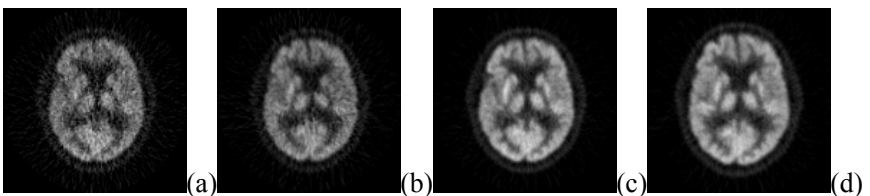

Figure 1. ADNI images: (a) a slice of original baseline images; (b) a coregistered image; (c) averaged image of dynamic scans; (d) reoriented image.

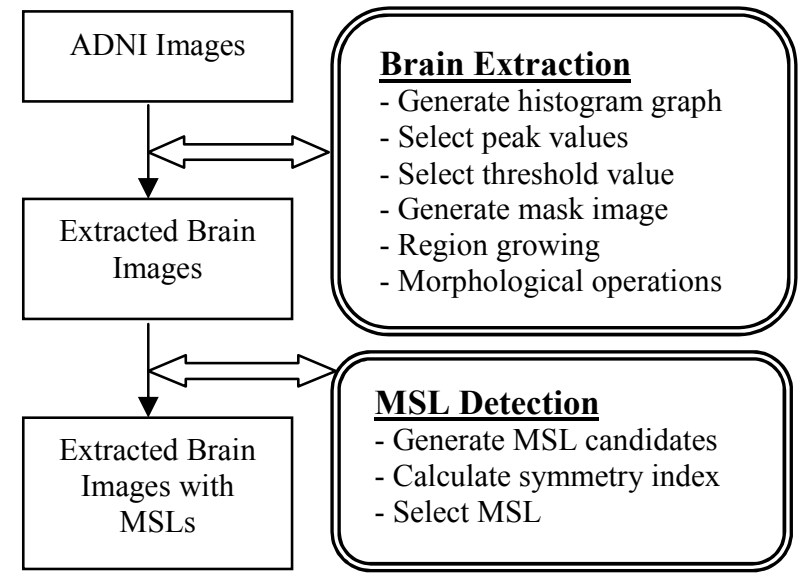

Figure 2. A flowchart of brain extraction and MSL detection.

\section{B. Method}

In FDG-PET images, the regions of the cerebrum and cerebellum are much brighter than the areas around or outside the crania. The proposed method automatically calculates an intensity value as a threshold to segment the images into brain and non-brain areas, followed by the MSLs detection on selected axial slices. The flowchart in Figure 2 shows the processing steps and the components of the brain extraction and the MSL detection. The brain extraction component is to: 1) generate a histogram graph from the volumetric dataset, 2) select two peak values and a threshold value from the graph, 3) generate the mask images based on the threshold value, and 4) apply region growing operation and morphological operations. The MSL detection component is firstly to generate the candidates of the MSL, and then calculate the symmetry index for each of them in order to finally select the MSL.

\section{Brain Extraction}

\section{1) Intensity histogram and peak points}

The histogram graph is created by counting the number of voxels with same intensity values in the whole volumetric images. Figure 3 shows the graphs with smoothed histogram by cutting off the highest peak of background for illustration purpose. Besides a highest peak of the image background, there are two situations about the peak points in the histogram graph: 1) two peaks to indicate the areas inside the crania and the areas around the crania (Figure 3a), 2) one peak to indicate the areas inside the crania (Figure $3 \mathrm{~b}$ ). For both situations, the peak points are selected by calculating the intensities gap $G$ at each intensity value. For example at the histogram graph of Figure 3 , let us consider $X$ as the horizontal axis of intensities and $Y$ as the vertical axis of the number of voxels which have same intensity values. The intensity gap value $G$ is defined as
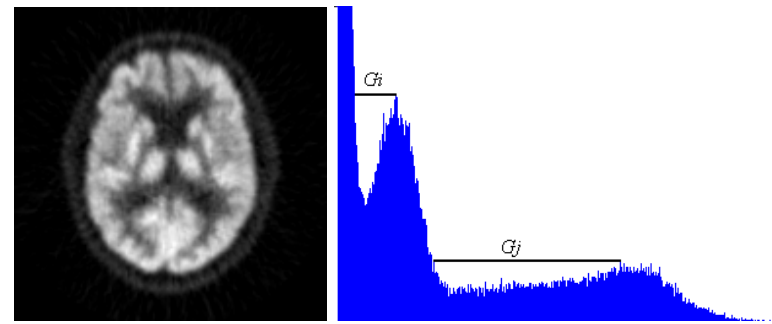

(a)
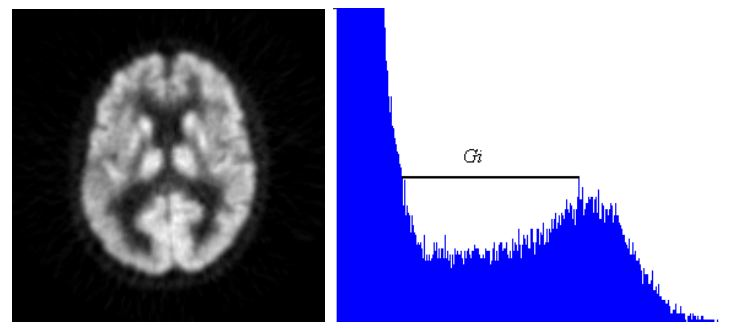

(b)

Figure 3. Histogram graphs: (a) with two peaks; (b) with one peak.

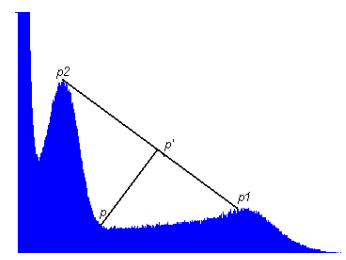

(a)

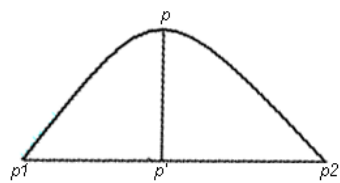

(b)

Figure 4. Selection of threshold value: (a) smoothed histogram graph, (b) highest point on the convex border.

$$
G_{i}=x_{i}-\max \left(x_{\mathrm{j}}\right) \text {, where } j<i \text { and } y_{j}=y_{i}
$$

where $i$ and $j$ are the positions on $X$ axis, $x_{i}$ is the intensity value of $i, y_{i}$ is the vertical height of $i$ on the histogram graph, $\max$ is to calculate the maximum value of all $x_{j}$ values which meet the defined conditions.

The points with larger $G$ values are the peaks of the graph. As the histogram graph has one peak or two peaks only, the points with the largest and the second largest $G$ values are selected as the peak points.

\section{2) Pick threshold value and generate binary images}

The peak point at the rightmost hand of the histogram graph (point $p_{1}$ in Figure 4a) indicates a class of pixels with higher intensity values, i.e. the pixels of brain cells. Another peak point $p_{2}$ indicates the non-brain or background pixels. In order to largely segment two classes of brain and non-brain pixels, the deepest point in the valley (point $p$ in Figure 4a) is selected as the threshold value. The point $p$ is also the highest point of the convex (Figure $4 \mathrm{~b}$ ), as well as the longest distance towards two peak points $\left(p_{1}\right.$ and $\left.p_{2}\right)$. To locate the highest point of convex, the orthogonal lines of the peak line $\left(L_{p 1 p 2}\right)$ are drawn to have two intersection points $p$ and $p^{\prime}$. The point $p$ is the intersection with the histogram graph, and the point $p^{\prime}$ is the intersection with $L_{p 1 p 2}$. The set of all the distances between $p$ and $p^{\prime}$, for all the points on the line $L_{p l p 2}$, is defined as

$$
D=\left\{d_{i}\left(p p^{\prime}\right), \quad x_{1}<i<x_{2}, \quad p^{\prime} \in L_{p 1 p 2}, \text { and } L_{p p^{\prime}} \perp L_{p 1 p 2}\right\}
$$



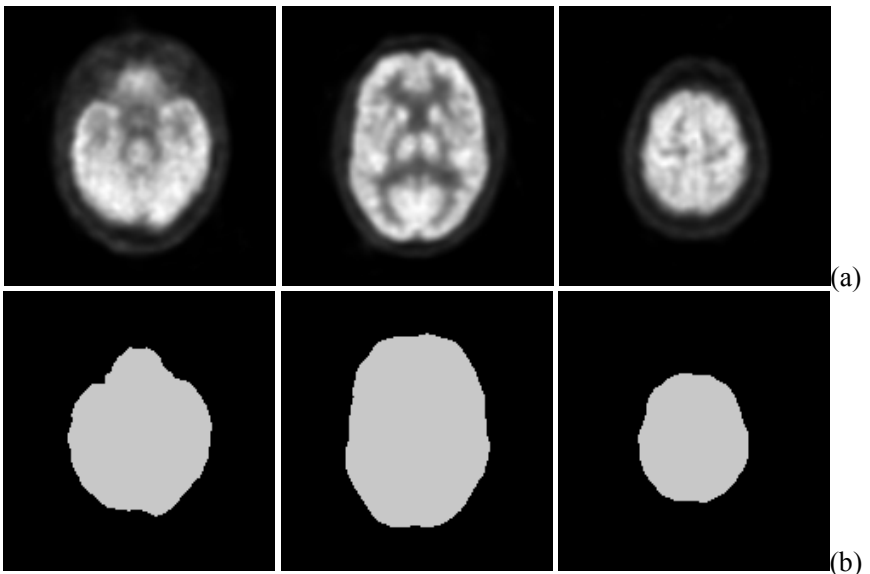

Figure 5. Extracted brain images: (a) original images; (b) extracted areas.

where $d_{i}\left(p p^{\prime}\right)$ is the distance between the points $p$ and $p^{\prime}, x_{1}$ and $x_{2}$ are the $x$-axis positions of point $p_{1}$ and $p_{2}, L_{p l p 2}$ and $L_{p p^{\prime}}$ are the lines of points $p_{1}$ and $p_{2}$, and points $p$ and $p^{\prime}$.

The corresponding $x$ value of the point, whose $d_{i}\left(p p^{\prime}\right)$ value is maximum in the set $D$, is the threshold value to segment the FDG-PET image into two parts: brain area and non-brain area. Based on the selected threshold value, the binary mask images are generated.

3) $3 D$ region growing and filling the holes inside the brain

Thresholding segmentation may leave some isolated points or small areas and may have holes inside the brain area. It is necessary to remove those isolated areas and fill those holes to complete the areas or volume inside crania. 3D region growing algorithm is applied to do so as the brain area is considered as a largest connected component. The seed point for region growing is the center of the volume. If it happens to be the nonbrain voxel, one of its brain voxel neighbors is automatically selected. The growing goes to three directions at the same time and complete until no more brain voxel added. Figure 5 shows the examples of three slices and their extracted areas from inferior slice (left) to superior slice (right).

\section{MSL Detection}

The brain areas are extracted and non-brain parts are removed from the FDG-PET images by applying the binary images generated from previous steps. Since the most inferior slices do not have detectable MSL, they are excluded from the calculation. About two-third superior slices of whole brain are selected for MSL detection. For example, if the most inferior slice is 5 and superior slice is 84 ( 80 slices in total), there 53 slices are calculated for MSLs from slice 30 to slice 82 .

We introduced earlier a symmetry index (SI) [12] for MSP detection from MR images. For detection MSL on FDG-PET images, it needs to be modified and extended to calculate the symmetry of brain cortex rather than the areas near the interhemispheric fissure, in order to avoid the areas (e.g. ventricles) which have less glucose uptake than brain cells. There are three steps to detect the MSL on an axial slice: 1) to choose the candidates, 2) to calculate the SI, and 3) to determine MSL.
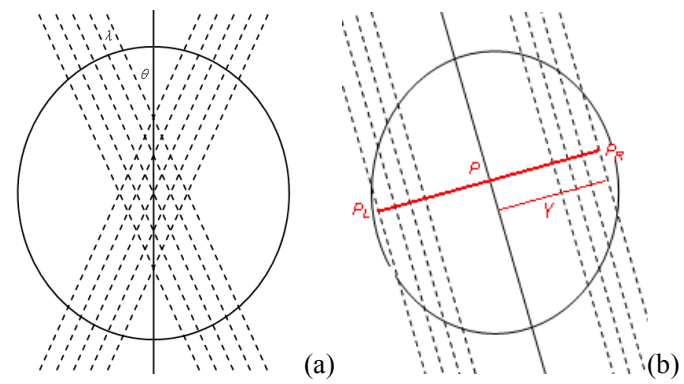

Figure 6. Detection of MSL: (a) the candidate lines; (b) calculation of SI.
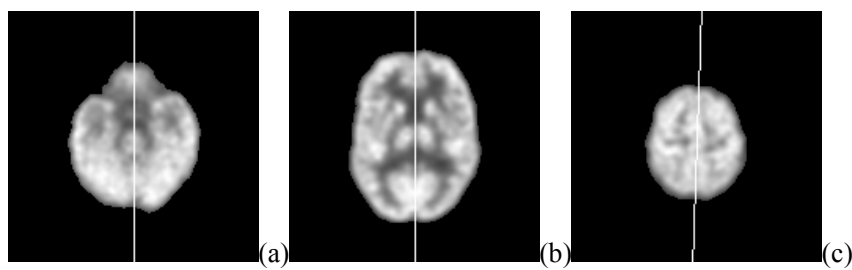

Figure 7. MSLs on: (a) inferior slice; (b) middle slice; and (c) superior slice.

\section{1) MSL candidates}

MSL is supposed to be an almost vertical line with some rotations and shifts. The range of rotations or shifts has their limits, e.g. the rotation not more than $40^{\circ}$. The candidates of MSL are chosen from those lines generated by the following step (Figure 6a):

1. the middle vertical line is the first candidate $(N v=1)$

2. rotate the middle vertical line to the left by $\theta$ degree (e.g. $\theta=2$ ) for $N r$ times (e.g. $N r=10$ )

3. rotate the middle vertical line to the right $\theta$ degree for $\mathrm{Nr}$ times

4. shift the line to the left by $\lambda$ pixels (e.g. $\lambda=1)$ for $N s$ times $(N s=10)$

5. shift the line to the right by $\lambda$ pixels for $N s$ times

A total of $N v+(2 \times N r) \times(2 \times N s)$ candidates are generated to calculate the SI.

\section{2) SI calculation}

For each candidate line (CL), SI is defined as an average of the sum of the difference between the left and the right of the CL. The calculation of SI is defined as (Figure 6b),

$$
S I(\theta, \gamma, k)=\sum_{1}^{H} \sum_{k=1}^{N c}\left|V\left(P_{L}\right)-V\left(P_{R}\right)\right| /\left(N_{c} \times H\right)
$$

where $H$ is the height of the image, $N c$ (e.g. $N c=5)$ is the number of pairs of the parallel lines which have same distance $(\gamma)$ to the CL. $V\left(P_{L}\right)$ and $V\left(P_{R}\right)$ are the intensity values of the mirrored points $P_{L}$ and $P_{R}$ on the parallel lines (Figure 6b). Their coordinates are defined as,

$$
P_{L, R}(x, y)=(P x \mp \gamma \cos \theta, P y \mp \gamma \sin \theta)
$$

\section{3) MSL selection}

The line with minimum SI from a group of candidates is selected as the MSL. Figure 7 shows the results of brain extraction and MSLs on an inferior slice, a middle slice and a superior slice. 
TABLE I. TEST RESULTS OF BRAIN EXTRACTION

\begin{tabular}{|c|c|c|c|c|c|}
\hline Case & $\begin{array}{c}\text { Manual } \\
\left.\mathbf{( c m}^{\mathbf{3}}\right)\end{array}$ & $\begin{array}{c}\text { Auto } \\
\left.\mathbf{( c m}^{\mathbf{3}}\right)\end{array}$ & $\begin{array}{c}\text { Sensitivity } \\
(\mathbf{\%})\end{array}$ & $\begin{array}{c}\text { Specificity } \\
(\mathbf{\%})\end{array}$ & $\begin{array}{c}\text { DI } \\
(\mathbf{\%})\end{array}$ \\
\hline 1 & 1143.86 & 1316.99 & 99.53 & 98.87 & 96.66 \\
\hline 2 & 1234.17 & 1393.21 & 99.91 & 97.73 & 93.86 \\
\hline 3 & 1305.84 & 1488.07 & 99.26 & 97.25 & 92.78 \\
\hline 4 & 1426.10 & 1709.43 & 99.96 & 95.87 & 90.93 \\
\hline 5 & 1443.07 & 1503.79 & 94.76 & 98.01 & 92.81 \\
\hline
\end{tabular}

TABLE II. TEST RESULTS OF MSL DETECTION

\begin{tabular}{|c|c|c|}
\hline Case & Angular difference $\mathbf{~}^{\mathbf{}}$ ) & Center displacement (mm) \\
\hline 1 & $1.09 \pm 0.87$ & $0.43 \pm 0.01$ \\
\hline 2 & $1.25 \pm 0.89$ & $0.41 \pm 0.01$ \\
\hline 3 & $0.87 \pm 0.48$ & $0.23 \pm 0.01$ \\
\hline 4 & $0.95 \pm 0.93$ & $0.58 \pm 0.15$ \\
\hline 5 & $1.47 \pm 1.12$ & $0.05 \pm 0.03$ \\
\hline
\end{tabular}

\section{RESULTS}

The proposed approach was implemented in $\mathrm{C}++$ running on a standard personal computer with $2.4 \mathrm{GHz} \mathrm{CPU}$. The average processing time was 3.03 seconds per case. A total of 226 cases with a resolution of $160 \times 160 \times 96$ voxels of size 1.5 $\mathrm{mm}^{3}$ were tested. Five of the datasets have the brain areas manually extracted and the MSLs manually drawn for comparison with the results of the automatic method. The other cases were visually checked with satisfactory results.

Table I provides the segmented results with sensitivity, specificity and Dice's index (DI). Sensitivity is the ratio of the number of true positives to the sum of true positives and false negatives. Specificity is the ratio of the number of true negatives to the sum of true negatives and false positives. DI is the radio of the overlap, between the automatically extracted areas and manually extracted areas, and the sum of them [17].

Table II provides the mean values and the standard deviations of the angular difference and the center displacements of comparing the automated MSL detection and the manual method. No significant statistical difference was observed. The angular difference $(D a)$ is defined as the angle between two lines $L, a x+b y+c=0$ a detected MSL line, and $L^{\prime}$, $a^{\prime} x+b^{\prime} y+c^{\prime}=0$ the line manually drawn (5). The center displacement $(D c)$ is defined as the distance of the center points of lines $L$ and $L^{\prime}(5)$.

$$
\begin{aligned}
& D a=\left|\operatorname{arccot}\left(-\frac{b}{a}\right)-\operatorname{arccot}\left(-\frac{b^{\prime}}{a^{\prime}}\right)\right| \times 180 \div \pi, \quad a \neq 0 \text { and } a^{\prime} \neq 0 \\
& D c=\left|\left(\frac{b \times H+2 \times c}{2 \times a}-\frac{b^{\prime} \times H+2 \times c^{\prime}}{2 \times a^{\prime}}\right)\right|, \quad a \neq 0 \text { and } a^{\prime} \neq 0
\end{aligned}
$$

where $H$ is the height of the image.

\section{DISCUSSION AND CONCLUSION}

A fast and fully automatic approach to extract the brain areas from FDG-PET images and detect MSLs from selected axial slices is presented. It has been applied to hundreds of cases and shown promising results. However, if the images have serious partial volume effect, those affected voxels are difficult to be classified into brain or non-brain areas. It may also face problems even for an experienced expert to manually segment the low resolution images like FDG-PET. To speed up the processing time, the proposed approach makes use of the histogram of the whole volume. However, for the cases with serious inhomogeneity between the slices, this method may cause false segmentation results. In such cases, the histogram of each slice may be helpful.

\section{ACKNOWLEDGMENT}

The data for this study have been obtained from the Alzheimer's Disease Neuroimaging Initiative (ADNI) database (www.loni.ucla.edu/ADNI). This research is partially supported by ARC DP0773584 and ARC LP0669645 grants.

\section{REFERENCES}

[1] M. Senda, Y. Kimura, and P. Herscovitch, Brain imaging using PET: Academic Press, 2002.

[2] R. A. Robb, D. P. Hanson, M. Found, and M. N. Rochester, "Analyze: A software system for biomedical image analysis," 1990, pp. 507-518.

[3] J. Mattout, C. Phillips, J. Daunizeau, and K. J. Friston, "Statistical parametric mapping: The analysis of functional brain analysis," London: Academic, pp. 367-76, 2007.

[4] J. M. Mykkanen, M. Juhola, and U. Ruotsalainen, "Extracting VOIs from brain PET images," International Journal of Medical Informatics, vol. 58-59, pp. 51-57, 2000.

[5] W. Koon-Pong, F. Dagan, S. R. Meikle, and M. J. Fulham, "Segmentation of dynamic PET images using cluster analysis," Nuclear Science, IEEE Transactions on, vol. 49, pp. 200-207, 2002.

[6] H. Koivistoinen, J. Tohka, and U. Ruotsalainen, "Comparison of pattern classification methods in segmentation of dynamic PET brain images," in Proceedings of the 6th Nordic Signal Processing Symposium NORSIG 2004, Espoo, Finland, 2004, pp. 73-76.

[7] K. N. Bhanu Prakash, Q. Hu, A. Aziz, and W. L. Nowinski, "Rapid and automatic localization of the anterior and posterior commissure point landmarks in MR volumetric neuroimages1," Academic Radiology, vol. 13, pp. 36-54, 2006.

[8] W. L. Nowinski, "Modified Talairach landmarks," Acta Neurochirurgica, vol. 143, pp. 1045-1057, 2001.

[9] W. L. Nowinski, "Computerized brain atlases for surgery of movement disorders," Seminars in Neurosurgery, vol. 12, pp. 183-194, 2001.

[10] W. L. Nowinski, A. Thirunavuukarasuu, and D. N. Kennedy, "Brain atlas for functional imaging: Clinical and research applications," Thieme, New York; Stuttgart, 2001.

[11] W. L. Nowinski, "Electronic brain atlases: Features and applications," in $3 D$ image processing: Techniques and clinical applications: Springer, 2002, p. 79.

[12] Q. Hu and W. L. Nowinski, "A rapid algorithm for robust and automatic extraction of the midsagittal plane of the human cerebrum from neuroimages based on local symmetry and outlier removal," NeuroImage, vol. 20, pp. 2153-2165, 2003.

[13] W. L. Nowinski, B. Prakash, I. Volkau, A. Ananthasubramaniam, and N. J. Beauchamp, "Rapid and automatic calculation of the midsagittal plane in magnetic resonance diffusion and perfusion images," Academic Radiology, vol. 13, pp. 652-663, 2006.

[14] I. Volkau, K. N. Bhanu Prakash, A. Ananthasubramaniam, A. Aziz, and W. L. Nowinski, "Extraction of the midsagittal plane from morphological neuroimages using the Kullback-Leibler's measure," Medical Image Analysis, vol. 10, pp. 863-874, 2006.

[15] F. P. G. Bergo, A. X. Falcao, C. L. Yasuda, and G. C. S. Ruppert, "Fast, accurate and precise mid-sagittal plane location in 3D MR images of the brain," Springer, 2009, p. 278.

[16] J. B. S. Langbaum, K. Chen, W. Lee, C. Reschke, D. Bandy, A. S. Fleisher, G. E. Alexander, N. L. Foster, M. W. Weiner, and R. A. Koeppe, "Categorical and correlational analyses of baseline fluorodeoxyglucose positron emission tomography images from the alzheimer's disease neuroimaging initiative (ADNI)," Neuroimage, vol. 45, pp. 1107-1116, 2009.

[17] L. R. Dice, "Measures of the amount of ecologic association between species," Ecology, pp. 297-302, 1945. 